\title{
Improving the Aquathermolysis Efficiency of Aromatics in Extra-Heavy Oil by Introducing Hydrogen-Donating Ligands to Catalysts
}

\author{
Ruilin Ren ${ }^{\mathrm{a}}$, Huachao Liu ${ }^{\mathrm{a}}, \mathrm{Yu}$ Chen ${ }^{\mathrm{a}}$, Jian $\mathrm{Li}^{\mathrm{b}}$,Yanling Chen ${ }^{\mathrm{a}, \mathrm{b}^{*}}$
}

\section{Supporting Information:}

In this paper, the mass balance of $\mathrm{N}$ and $\mathrm{S}$ were calculated according to the equation $\mathrm{Nt}=$ Wc*Mc. (where Wc is the group compositions' mass fraction, and Mc is the N, S elements' content in group compositions, so we get the total content (Nt) per 10000g oil samples). Table S1 and Table S2 show the results of $\mathrm{N}$ and S elements' content in aromatics, resins, and asphaltenes of oil samples before and after reaction. Saturates were ignored, for the reason that the separation of the four group compositions depended on the polar property.

Fig. S1 is a separation flowchart of four components in heavy oil.

Fig. S2 is ${ }^{1}$ HNMR spectra of aromatic compositions.

Dimethylbenzenesulfonic copper was used as a catalyst. In order to avoid the impact of the product which were derived from the catalyst after aquathermolysis, a experiment was designed to exclude the impact of catalyst. In the absence of heavy oil, the hydrothermal reactions of the catalyst was carried out at $240{ }^{\circ} \mathrm{C}$ for $24 \mathrm{~h}$, and then, the product was detected with GC-MS (Fig. S3). The results (Table S3) showed that the hydrocarbons what we discussed in the paper did not derive from the catalyst but heavy oil. The date of Table S3 is corresponding to Fig.S3.

Fig. S4 is the thermal stability characterization of the as-prepared catalyst 


\section{Table and Fig.:}

Table S1. Nitrogen content of aromatics, resins and asphaltenes in different oil samples

Table S2. Sulfur content of aromatics, resins and asphaltenes in different oil samples

Table S3. The identification of products after the hydrothermal reaction of the catalyst.

Fig. S1. The separation flowchart of four components in heavy oil

Fig. S2. ${ }^{1}$ HNMR spectra of aromatic compositions (a. ${ }^{1}$ HNMR spectra of aromatic hydrocarbons in crude oil sample I b. ${ }^{1} \mathrm{HNMR}$ spectra of aromatic hydrocarbons in oil sample II, c. ${ }^{1} \mathrm{HNMR}$ spectra of aromatic hydrocarbons in oil sampleIII.)

Fig. S3. Total ion chromatogram of products after the hydrothermal reaction of catalyst.

Fig. S4. Thermal stability characterization of the as-prepared catalyst. 
Table S1 Nitrogen content of aromatics, resins and asphaltenes in different oil samples

\begin{tabular}{ccccc}
\hline \multirow{2}{*}{ Oil sample } & \multicolumn{3}{c}{ N content } & Total \\
\cline { 2 - 4 } & Aromatics & Resins & Asphaltenes & content \\
\hline Oil sample I & 19.039 & 58.708 & 50.556 & 128.303 \\
Oil sample II & 20.299 & 60.051 & 44.025 & 124.375 \\
Oil sample III & 24.698 & 51.345 & 42.075 & 118.118 \\
\hline
\end{tabular}


Table S2 Sulfur content of aromatics, resins and asphaltenes in different oil samples

\begin{tabular}{ccccc}
\hline \multirow{2}{*}{ Oil sample } & \multicolumn{3}{c}{ S content } & \multirow{2}{*}{ Total } \\
\cline { 2 - 4 } & Aromatics & Resins & Asphaltenes & content \\
\hline Oil sample I & 65.898 & 59.354 & 62.669 & 187.922 \\
Oil sample II & 70.285 & 65.346 & 52.389 & 188.019 \\
Oil sample III & 82.528 & 56.868 & 48.019 & 187.415 \\
\hline
\end{tabular}


Table S3. The identification of products after the hydrothermal reaction of the catalyst.

\begin{tabular}{cccc}
\hline Figure & Time(min) & Chemical formula & Compounds \\
\hline $\mathrm{S} 1$ & 9.21 & $\mathrm{C}_{13} \mathrm{H}_{10} \mathrm{O}$ & benzophenone \\
\hline
\end{tabular}

The date of Table S3 is corresponding to the Fig. S3. 
Fig. S1. The separation flowchart of four components in heavy oil

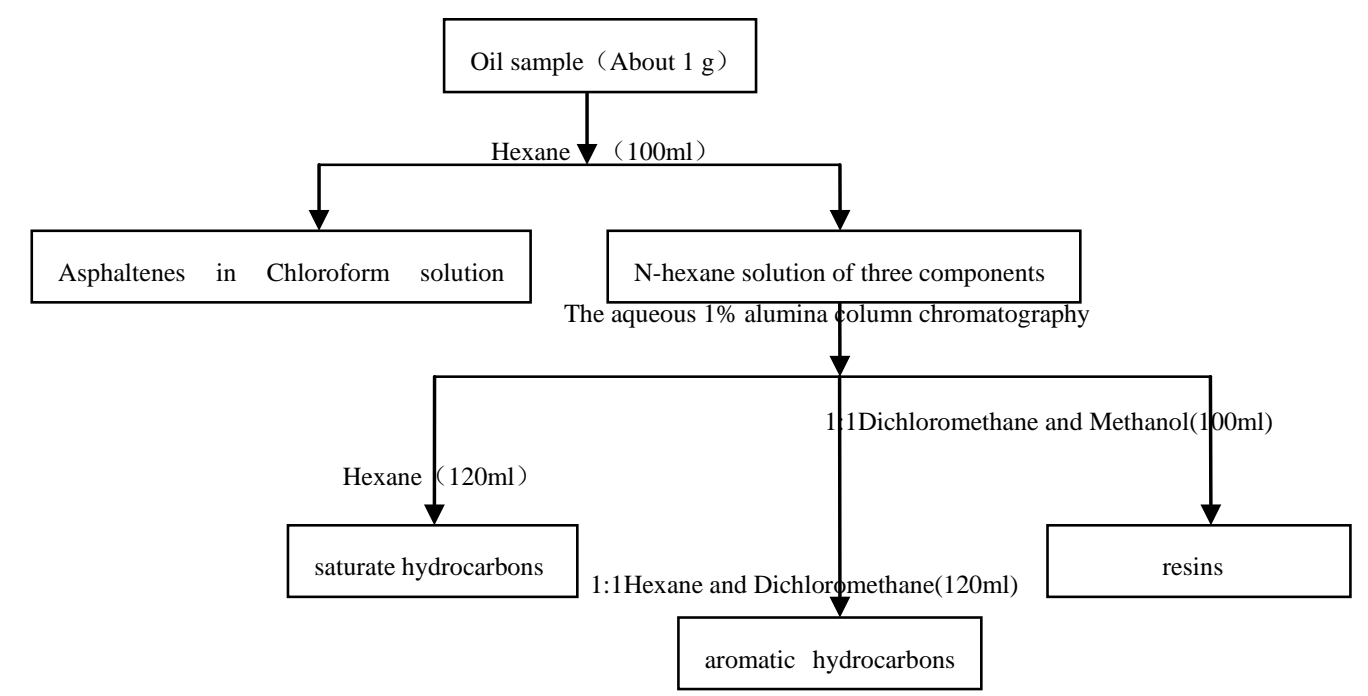


Fig. S2. ${ }^{1}$ HNMR spectra of aromatic compositions (a. ${ }^{1}$ HNMR spectra of aromatic hydrocarbons in crude oil sample I ,b. ${ }^{1} \mathrm{HNMR}$ spectra of aromatic hydrocarbons in oil sample II, c. ${ }^{1} \mathrm{HNMR}$ spectra of aromatic hydrocarbons in oil sampleIII.)

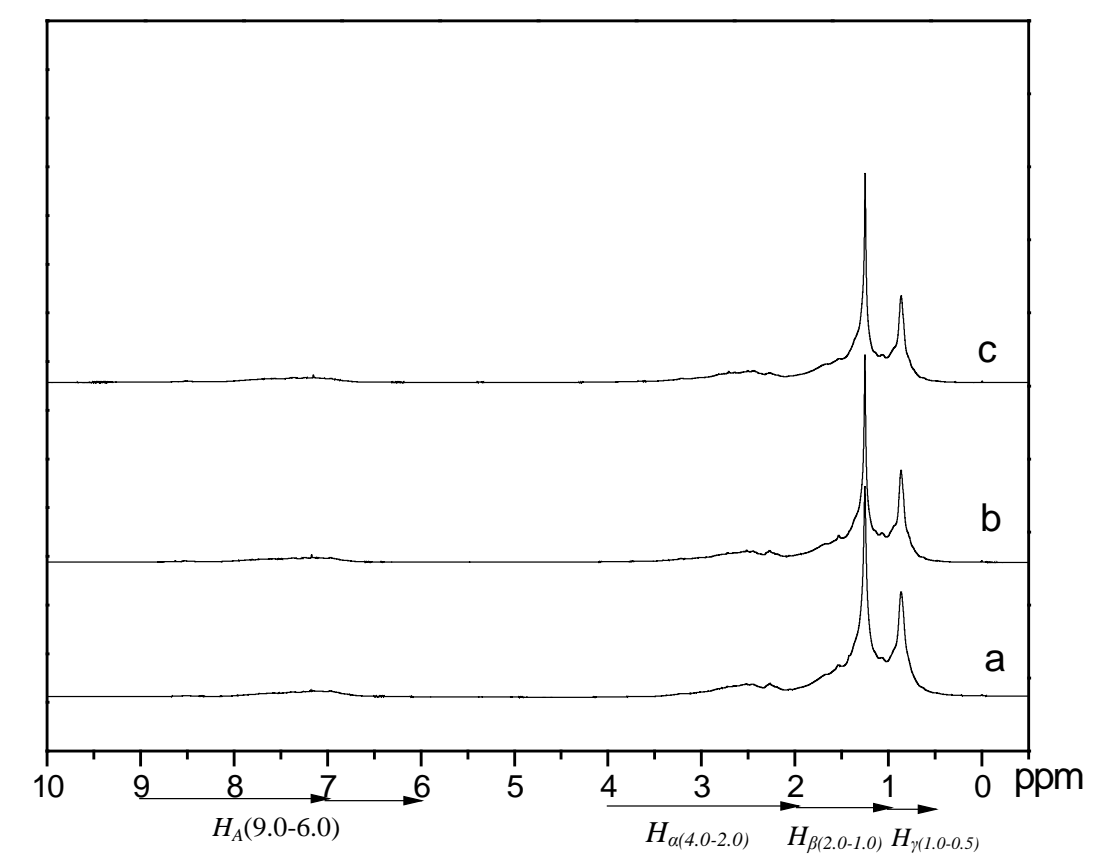


Fig. S3. Total ion chromatogram of products after the hydrothermal reaction of catalyst.

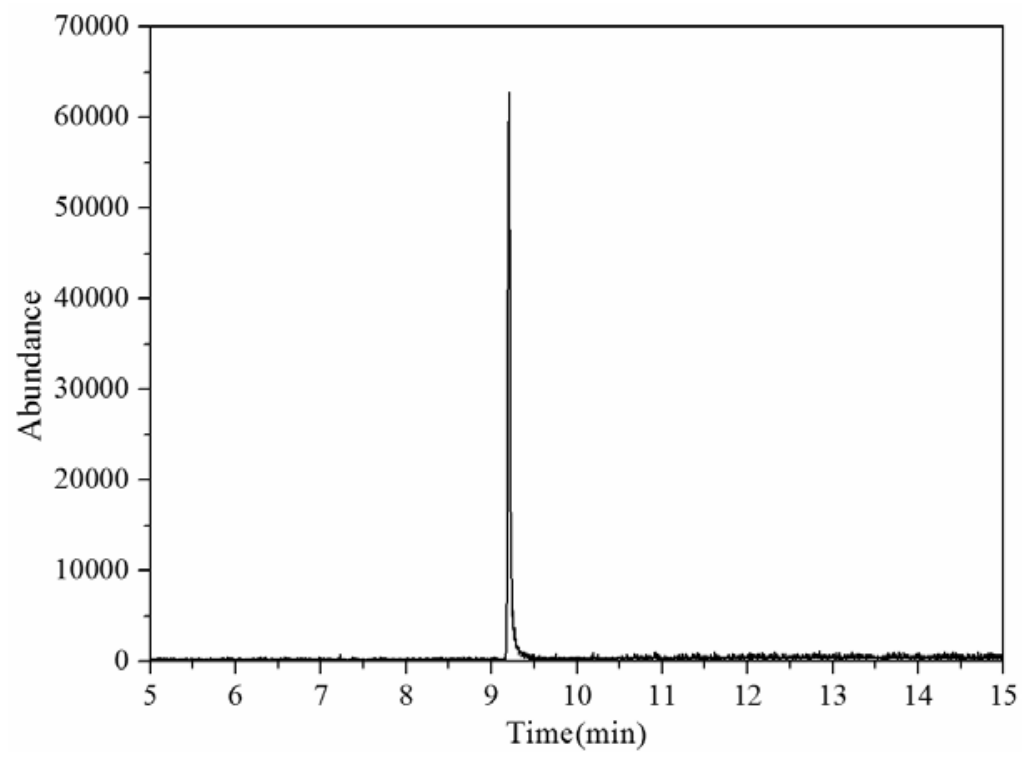


Fig. S4. Thermal stability characterization of the as-prepared catalyst.

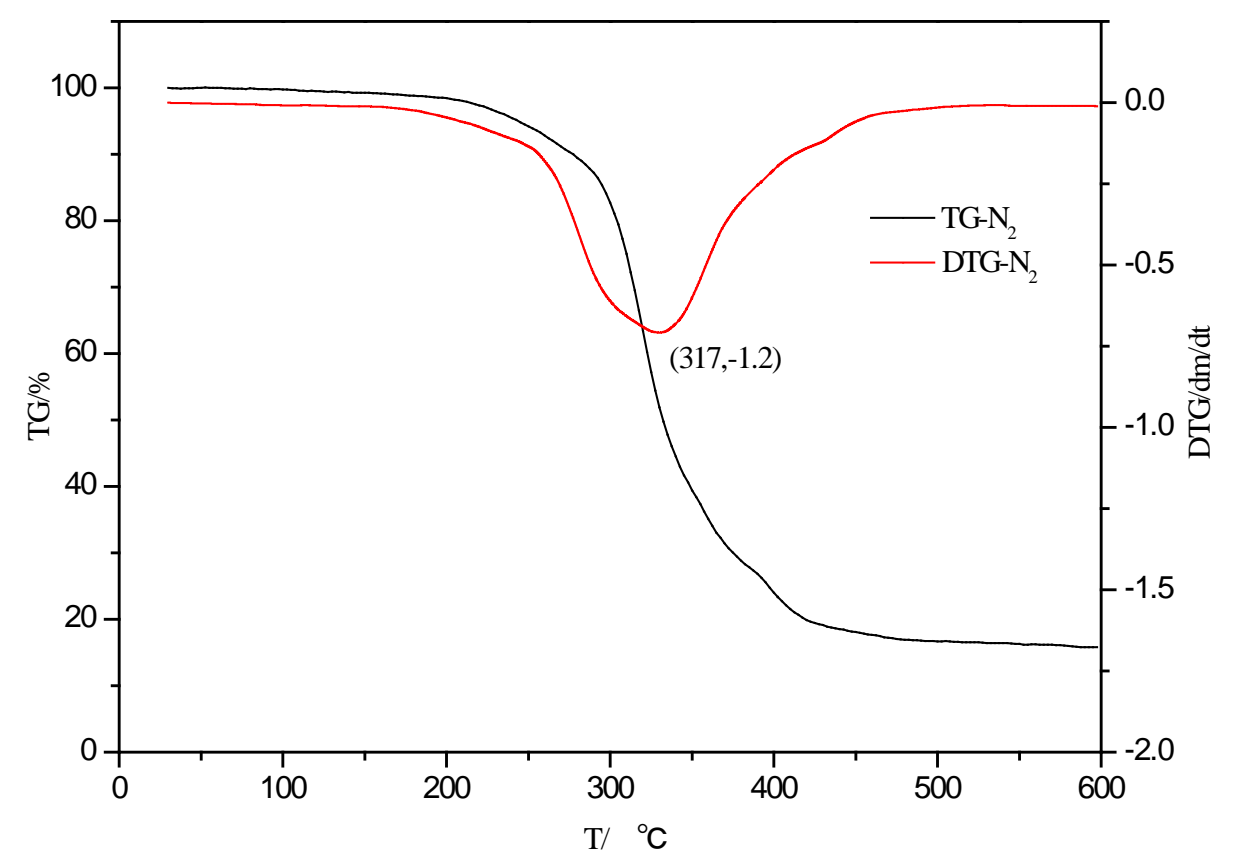


Equations:

$$
\begin{aligned}
& f_{A}=\frac{C_{T} / H_{T}-\left(H_{\alpha}+H_{\beta}+H_{\gamma}\right) / 2 H_{T}}{C_{T} / H_{T}} \\
& \frac{H_{A U}}{C_{A}}=\frac{H_{A} / H_{T}+H_{\alpha} / 2 H_{T}}{C_{T} / H_{T}-\left(H_{\alpha}+H_{\beta}+H_{\gamma}\right) / 2 H_{T}} \\
& B I=\frac{\frac{1}{3} H_{\gamma}}{\frac{1}{2}\left(H_{\alpha}+H_{\beta}\right)} \\
& \sigma=\frac{C_{\alpha}}{C_{A P}}=\frac{H_{\alpha} / 2}{H_{A}+H_{\alpha} / 2}
\end{aligned}
$$

$f_{A}$ : the aromaticity of aromatic hydrocarbons

$H_{A U} / C_{A}$ : the aromaticity condensation of aromatic hydrocarbons

$B I$ : branching index of aromatic hydrocarbons

$\sigma$ : substitution rate of aromatic ring

$H_{A}(9.0-6.0), H_{\alpha}(4.0-2.0 \mathrm{ppm}), H_{\beta}(2.0-1.0 \mathrm{ppm})$, and $H_{\gamma}(1.0-0.5 \mathrm{ppm})$, they represent different chemical shifts of attributable hydrogen. 